\title{
Identification of MHD Mode Structure using ECE and SX Measurements* $^{*}$
}

\author{
Hayato TSUCHIYA, Yuuki TAKEMURA ${ }^{1)}$, Yoshio NAGAYAMA, Daisuke KUWAHARA ${ }^{2)}$, \\ Kiyomasa WATANABE, Satoru SAKAKIBARA and LHD Experimental Group \\ National Institute for Fusion Science, Toki 509-5292, Japan \\ ${ }^{1)}$ The Graduate University for Advanced Studies, Toki 509-5292, Japan \\ ${ }^{2)}$ Tokyo Institute of Technology, Meguro-ku, Tokyo 152-8550, Japan
}

(Received 9 December 2011 / Accepted 4 March 2012)

\begin{abstract}
This study reports characteristics of the interchange mode-like magnetohydrodynamic (MHD) mode through comparisons between fluctuations measured by electron cyclotron emission (ECE) and Soft X-ray (SX) in the Large Helical Device (LHD). The MHD mode, whose frequency is 0.74 to $2.7 \mathrm{kHz}$, is enhanced near $\rho=0.8, \iota=$ 1. $T_{\mathrm{e}}$ fluctuation data measured by ECE can identify the mode width by using the displacement amplitude profile whereas SX data cannot show the structure clearly. Furthermore the dependence of the width and amplitude on the pressure gradient is shown using $T_{\mathrm{e}}$ fluctuations.
\end{abstract}

(c) 2012 The Japan Society of Plasma Science and Nuclear Fusion Research

Keywords: MHD low-order mode, LHD, electron cyclotron emission, soft x-ray, displacement amplitude

DOI: $10.1585 /$ pfr.7.2402056

\section{Introduction}

It is well known that edge resonant low-order modes affect radial structures such as local flattening in density or temperature profiles, which may cause degradation in plasma performance. In the Large Helical Device (LHD) [1], some magnetohydrodynamic (MHD) modes, including the interchange-type instability, excited in the edge region of a torus plasma have been observed in the high-beta regime [2], which does not lead to disruptive discharge termination. However, the instability is considered to be related to the decision mechanism of the achieved achieved beta values [3]. Therefore, it is quite important to obtain the data of magnetic fluctuations and instability mode structures, which are basic data for the study and control of the MHD instability [4].

The fluctuation study is usually performed based on the data of magnetic fluctuations and soft X-ray (SX) intensity fluctuations. Magnetic fluctuations can be identified by the mode number by using a multi-magnetic probe (MP), but cannot directly give information on the mode structure because the observed magnetic fluctuation is a global value. In addition, the SX intensity is also determined by a line integral value along the sight line. Therefore, it is difficult to convert the line-integrated fluctuation data into a local fluctuation value. The results of the SX fluctuation profile are inadequate to specify the location of the fluctuation. In this paper, the characteristics of the mode are investigated using the magnetic probe, SX and electron cyclotron emission (ECE) [5], which is good

author's e-mail: tsuchiya.hayato@lhd.nifs.ac.jp

*) This article is based on the presentation at the 21 st International Toki Conference (ITC21). for identifying the measurement location. In addition, the comparison between the sensitivity of MP and ECE is reported.

\section{Experimental Setup}

This study was performed at the LHD [1]. The MHD fluctuations were measured mainly by MP [6], SX detector arrays [7] and radiometer of ECE [5]. The frequency resolution of ECE is $1 \mathrm{GHz}$. It corresponds to a spatial resolution of $\Delta \rho \sim 0.02$ at $\rho \sim 0.8 B_{\mathrm{ax}}=1.5 \mathrm{~T}$, where $\rho$ is the normalized radius. MHD or high-beta experiments are often conducted in conditions of low magnetic field $B_{\mathrm{ax}}<$ $1.0 \mathrm{~T}$ to clarify the effect on the magnetic field. Under these conditions, ECE measurements cannot be used because of the cutoff at the LHD. Instead, SX radiation measurement is a helpful tool for fluctuation studies. The spatial resolution of the SX detector array is approximately $3 \mathrm{~mm}$ $(\Delta \rho \sim 0.06)$ at mid plane. The experiment is conducted under the condition of $R_{\mathrm{ax}}=3.6 \mathrm{~m}, B_{\mathrm{ax}}=1.5 \mathrm{~T}$, which is suitable for measuring ECE in the range $0.1<\rho<1$. Figure 1 shows the SX array sight line, the magnetic configuration, and the ECE measurement point for these conditions. The SX sight line and ECE sight line are not on the same cross section. The SX array is installed at the upper port in the vertical cross section (Fig. 1 a)). The SX measurement system consists of two of silicon PIN photodiodes arrays [7]. The chords of the one of the arrays pass the torus-outboard side and those of the other array pass the torus-inboard side. Meanwhile, the antenna of ECE is installed at the outer side port in the horizontal cross section. Because the magnetic configuration of the LHD results in 

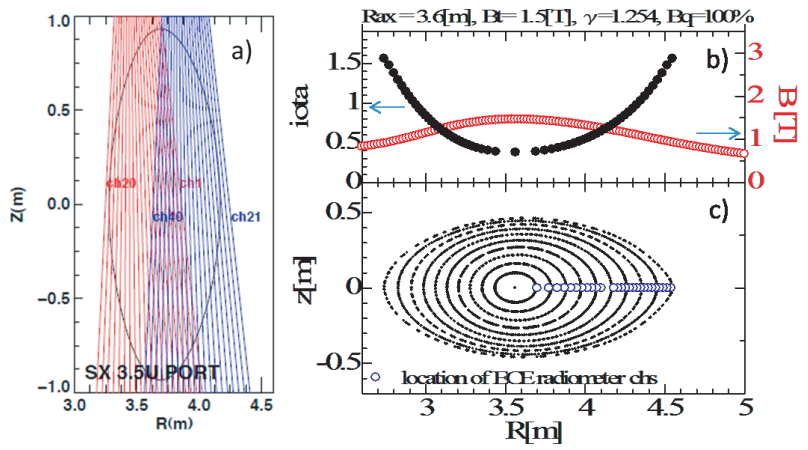

Fig. 1 a) SX sight line. The detector array is installed at the upper port. b) The magnetic configuration of LHD. The rotational transform $\iota$ profile (left axis) and magnitude of the magnetic field (right axis) along the ECE sight line. c) Location of ECE measurement points. The ECE antenna is installed at the side port. Frequency resolution of ECE is $1 \mathrm{GHz}$.

a peak magnetic field around the magnetic axis in the cross section (as shown in Fig. 1 b)), the outer side of torus is the measurement area of ECE. Figure $1 \mathrm{c}$ ) shows the measurement points of available channels including the $\iota \sim 1$ area $(R \sim 4.35 \mathrm{~m})$. The frequency range of second harmonics of the ECE X-mode is 53-84 GHz. When we compare the location between these measurements, the normalized radius $\rho$ is usually used. If the identified mode structure used by SX is supported by the ECE measurement, this study would help the confinement studies of the low-field discharge.

\section{Experimental Results}

\subsection{Characteristic of low-order MHD mode}

The MHD fluctuations of the low-order mode were observed in a discharge heated by tangential neutral beam injection (NBI : co-NBI 3.8 MW, counter-NBI 10.5 MW). In the discharge, shown in Fig. 2, the line-averaged density does not change during $t=3.5$ to $5.0 \mathrm{~s}$ and the plasma current increases gradually by counter-NBI, which makes for a strong magnetic shear. Fig. 2 d) shows the development of the power spectrum of the magnetic fluctuation. We can see the significant power peak at $f=0.7$ to $2.8 \mathrm{kHz}$ driven by the MHD instability, which is $m=1 / n=1$ where $m$ and $n$ are, respectively, the poloidal and toroidal mode numbers of the MHD instability.

The radial-fluctuation power peaks in the ECE and SX at $\rho \sim 0.85$, which corresponds to the area of $\iota=1$. Figure 3 shows the coherence evolution between ECE, SX and MP measurements. We can see the existence of a broad peak from 1.9 to $2.7 \mathrm{kHz}$, and the peak frequency gradually decreases with the increasing of toroidal currents. We consider that these fluctuations originate from the same instability.

Multi-channel Fourier analysis for the $f=1.9$ to $2.7 \mathrm{kHz}$ fluctuation components at $t=3.82 \mathrm{~s}$ show not only

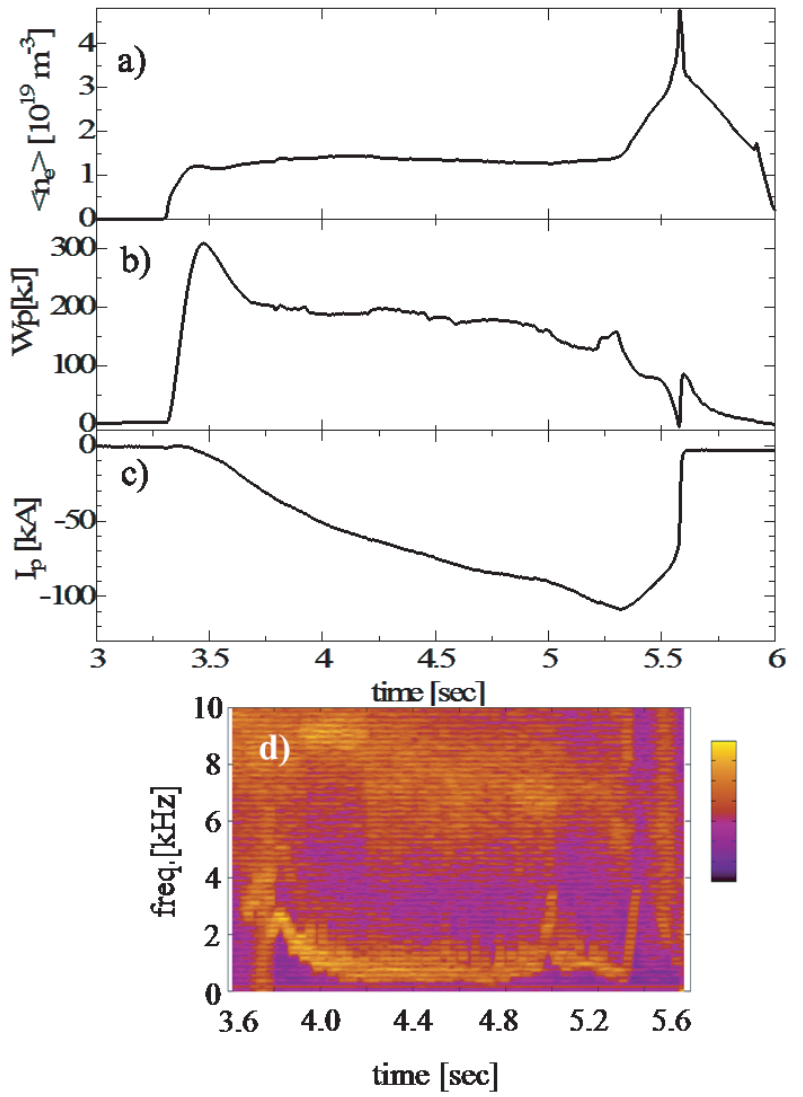

Fig. 2 Discharge waveform a) development of electron lineaveraged density $\left\langle n_{\mathrm{e}}\right\rangle, \mathrm{b}$ ) development of the stored energy $W_{\mathrm{p}}$, c) development of toroidal plasma current, and d) spectrogram of magnetic probe data.

that there is a peak in the radial fluctuation level profile but also that there is a peak in the radial coherence profile between neighboring channels. In particular, there is high coherence at $\rho=0.75$ to 0.9 . In the area, $T_{\mathrm{e}}$ oscillates in phase, and the phase profile of SX fluctuations shown in Fig. 4 a) indicates a similar tendency. We consider that these indicate that the MHD mode have finite width. That the inboard side and outboard sides are out of phase supports $n=1$. We compare the results of the coherence profile calculated from ECE and SX fluctuation data. To take into account the broad power peak, the coherence shown in Fig. 4 b) is the average of the coherence of $f=$ 1.9 to $2.7 \mathrm{kHz}$. The fluctuations excited around $\rho=0.85$ seem to reach $\rho=0.6$. The coherence calculated from SX data tends to be larger than that calculated from ECE data, which we attribute to the SX intensity including the information of passing each magnetic surface along the line of sight, so care should be taken in case the identification of location using SX fluctuation.

To clarify the mode structure, we calculate the displacement amplitude $\xi$. The displacement amplitude is defined as

$$
\xi=\frac{-\delta g}{\delta \bar{g} / \delta \rho},
$$




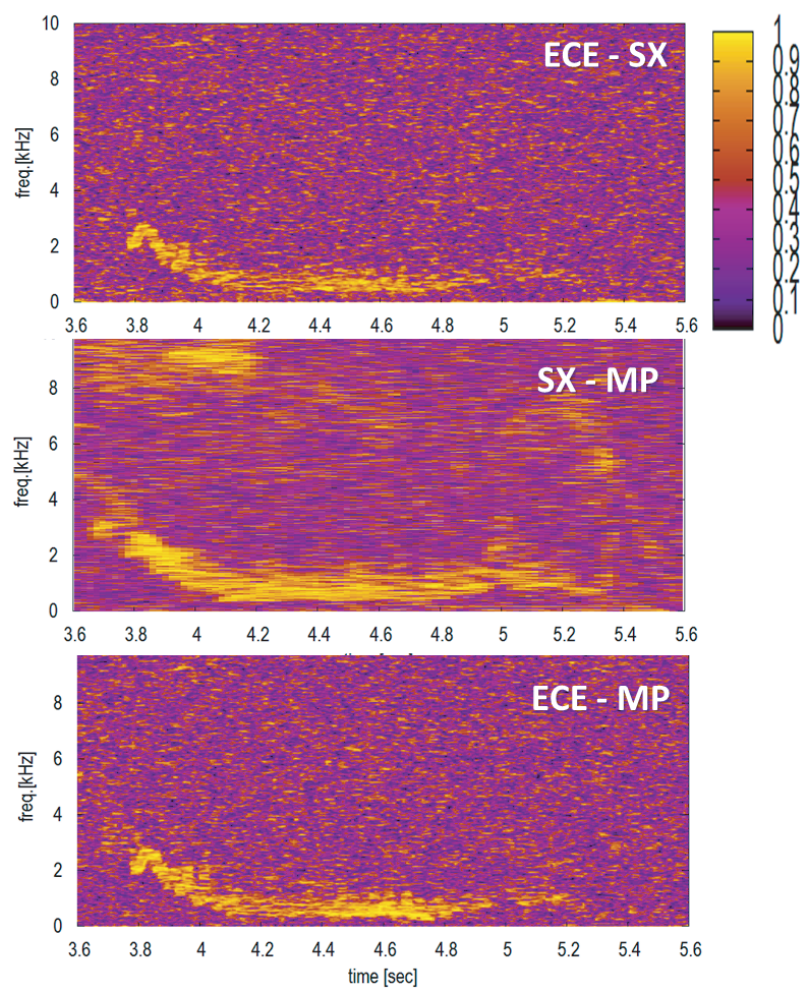

Fig. 3 Coherence evolution among ECE, SX and MP measurements. The measurement point of both ECE and SX is $\rho \sim 0.85 . n_{\mathrm{e}} \sim 1.5 \times 10^{19} \mathrm{~m}^{-3}, T_{\mathrm{e}} \sim 1.5 \mathrm{keV}$.

where, $g$ is the measured physical value. In our case, $g$ is SX intensity or ECE intensity. $\bar{g}$ is the time averaged value of fluctuation data, (i.e., the DC component) of the SX or ECE intensity. In the strict sense, the line-integrated SX intensity has to be converted to the local value by the Abel inversion method. The displacement amplitude using SX intensity is calculated without the conversion. The value of $\delta \bar{g} / \delta \rho$ is numerically derived from the derivative of the fitting function of each intensity profile.

Figure $4 \mathrm{c}$ ) compares the displacement amplitude profile using the ECE and SX fluctuation data. When the SX signal (white mark) cannot show the structure clearly, we can see the mode structure using the ECE (filled marks). There are cases where the significant value is calculated based on the SX data [2]. The pressure profile, also shown in Fig. 4 d), is a hollow profile, and has a negative gradient coefficient around the $\iota \sim 1$ surface. Considering the facts as previously described in this section, we estimate that the MHD mode is excited by the interchange instability.

Because the interchange instability is driven by the pressure gradient, there should be a relationship between the mode structure and the pressure gradient. Figure 5 summarizes the dependence of mode width and displacement amplitude on the pressure gradient. Applying a Gaussian fit to the displacement amplitude profile, we estimate the width of the mode to be the half bandwidth of the Gaussian. We can thus clearly evaluate dependence of the mode

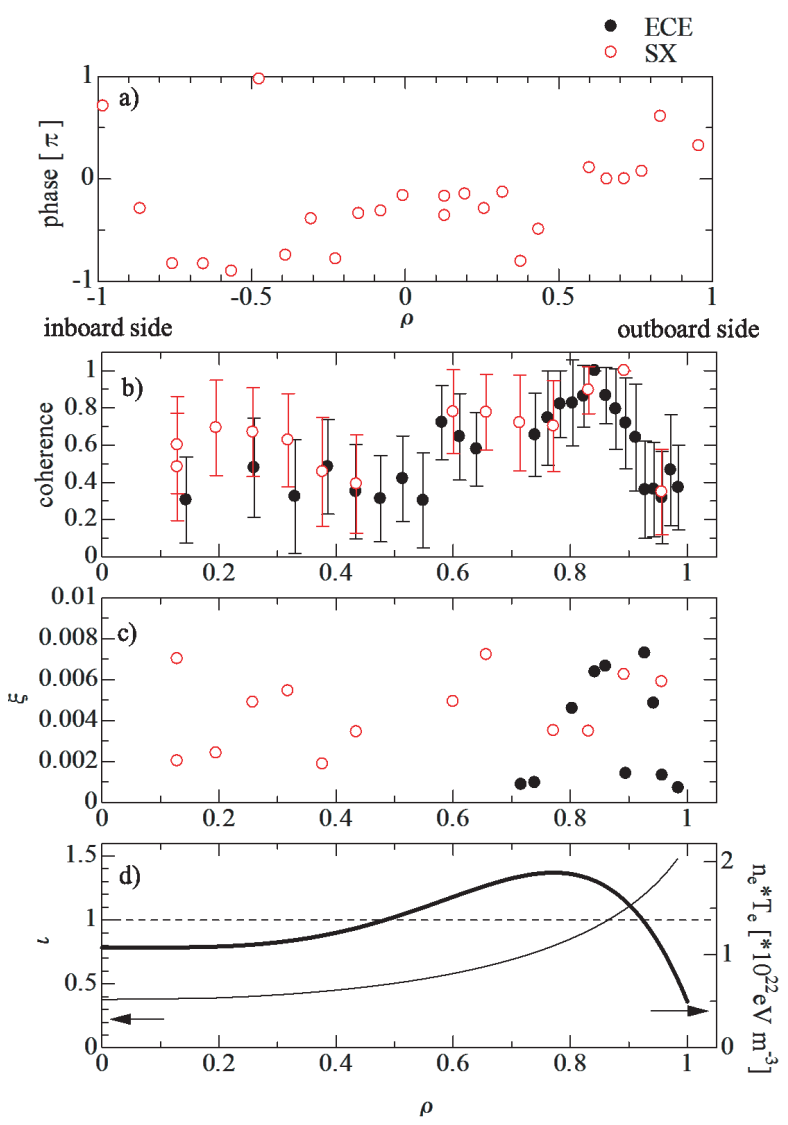

Fig. 4 a) Phase profile of $f=2.5 \mathrm{kHz}$ fluctuation calculated based on SX data. Comparison of the coherence profile b) and $\xi$ profile c) between ECE and SX. $f=1.9$ to $2.7 \mathrm{kHz}$. d) Iota profile calculated in vacuum condition and pressure profile calculated from Thomson-scattering data.

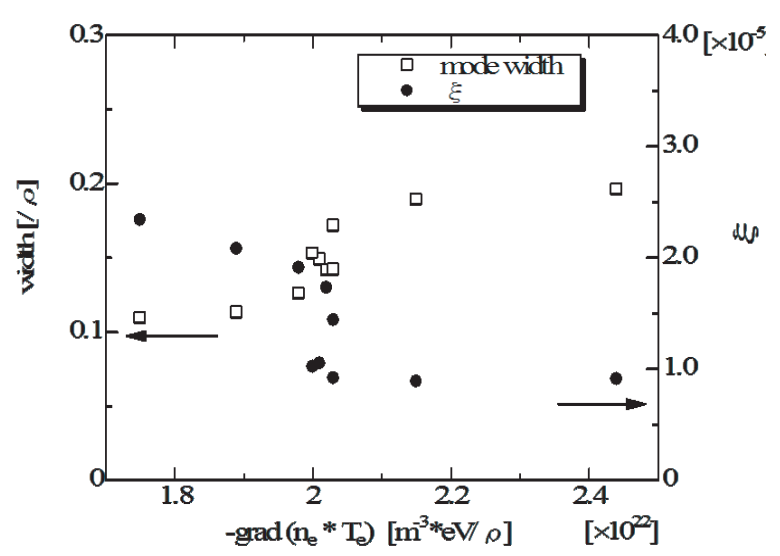

Fig. 5 Dependence of mode width (left axis) and $\xi$ (right axis) on pressure gradient.

width and displacement amplitude on the pressure gradient. We find that the mode width widens with increasing pressure gradient (white point in Fig. 5). The displacement amplitude increases less strongly with the pressure gradient (filled marks). 


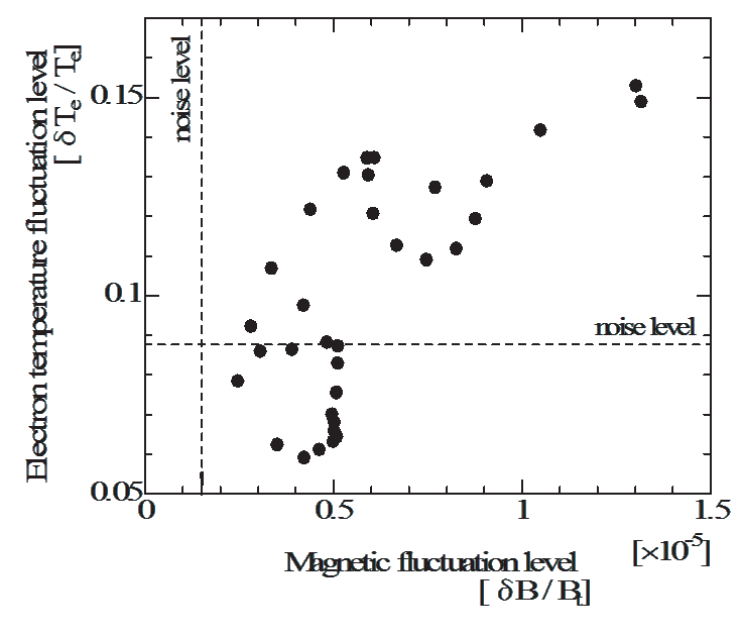

Fig. 6 Relationship between magnetic and electron temperature fluctuation level calculated from MP and ECE data.

\subsection{Comparison of sensitivity for MHD mode between MP and ECE}

The MHD mode is clearly and easily detected by MPs. The MHD mode observed by MP cannot often be observed by ECE as an electron temperature fluctuation. If the oscillation amplitude is small, the electron temperature calculated by the ECE data would be under the noise level. Figure 6 shows the sensitivity of MP and ECE. Both noise levels are also drawn as horizontal or vertical dashed lines in the figure. The noise level is evaluated by integrating the fitting power spectrum without the characteristic power peak. The electron temperature fluctuation level increases with the magnetic fluctuation, as expected. Because the magnetic oscillation is small, the measured temperature fluctuation of every ECE channel, derived from some MHD instabilities, is likely caused by the oscillation of the magnetic surface. In this sense, generally, largeamplitude electron temperature fluctuations are not generated when the radial temperature gradient is small, even if there is radial pressure gradient.

\section{Summary and Discussions}

By using SX and ECE data, we have analyzed the low- order MHD mode structure $(m=1 / n=1)$ excited at the $\iota \sim 1$ surface and whose frequency is 0.7 to $2.8 \mathrm{kHz}$. Both measurements show the localized mode as a radial coherence profile. When discussing the radial characteristics of fluctuations using SX, we note the results of the core chord SX because they include the effect of fluctuations in the surrounding area. The coherence profile obtained from SX data agrees with the tendency of ECEs at surrounding area, though the coherence values of the SX data is globally larger than those of ECE. These facts indicate that the ECE supports the reasonability and availability of the analysis of SX fluctuations even for low-magnetic-field conditions where ECE measurement is not available. The large-amplitude fluctuation structure can be described by ECE data as the displacement amplitude. The number of ECE fluctuation data is limited due to the low sensitivity of ECE. It is issue to store the data of various conditions, and we have to understand the exact relationship between results of ECE and SX without an Abel inversion. These results and the associated stability analysis should clarify the decision mechanism for the achieved high beta value.

\section{Acknowledgments}

The authors thank the LHD experimental group for their technical support. The work is supported by the budget NIFS11ULPP008 and NIFS11ULPP020 of the National Institute for Fusion Science.

[1] O. Motojima et al., Nucl. Fusion 40, 599 (2000).

[2] K.Y. Watanabe, S. Masamune, Y. Takemura et al., Phys. Plasmas 18, 056119 (2011).

[3] J.H. Harris, M. Murakami, B.A. Carreras, J.D. Bell et al., Phys. Rev. Lett. 63, 1249 (1989).

[4] S. Masamune, 23rd IAEA Fusion Energy Conference, Daejon, Republic of Korea, EXS/P5-11.

[5] H. Tsuchiya, Y. Nagayama, K. Kawahata, S. Inagaki, S. Kubo and LHD Experiment Group, Plasma Fusion Res. 6, 2402114 (2011).

[6] S. Sakakibara, H. Yamada, K. Yamazaki, K.Y. Watanabe et al., Fusion Eng. Des. 34-35, 707 (1997).

[7] S. Ohdachi, F. Watanabe, S. Yamamoto, K. Toi, C. Suzuki, K. Ida, S. Muto and LHD Experiment Group, Fusion Sci. Technol. 58, 418 (2010). 